\title{
Luminescence of the pyrazoline dye in nanostructured zeolite matrix
}

\author{
I.V. Beliak', V.G. Kravets ${ }^{2}$, A.A. Kryuchin ${ }^{3}$ \\ Institute for Information Recording, NAS of Ukraine (IPRI NASU) \\ 2, M. Shpak str., 03113 Kyiv, Ukraine \\ ${ }^{1}$ Phone: (38-044) 454-22-09, fax: (38-044) 241-72-33; e-mail: festiv@yandex.ru \\ 2Phone: (38-044) 454-21-90,fax: (38-044) 241-72-33; e-mail:vasyl_kravets@yahoo.com \\ ${ }^{3}$ Phone: (38-044) 454-21-52, fax: (38-044) 241-72-33; e-mail: ipri@ipri.kiev.ua
}

\begin{abstract}
A physicotechnical fundamental of the multilayer photoluminescent media development has been considered. The quantum yield and relaxation time of luminescence were found as most significant values of the recording material. Organic pyrazoline dyes were adopted due to their appropriable characteristics. Possibility of the further luminescent material parameters increase while performance dye molecules in the white nanostructured zeolite matrix by components mixing and laser annealing was proposed. Experimental results with $60 \%$ of quantum yield gain and relaxation time quadruple decrease was obtained. Using the received materials in plotting the identification luminescent elements based on the optical disc cover procedure has been determined.
\end{abstract}

Keywords: multilayer photoluminescent media, quantum yield, relaxation time, pyrazoline dyes, zeolite, nanostructured matrix, identification elements.

Manuscript received 19.12.06; accepted for publication 26.03.07, published online 01.06.07.

Nowadays organic luminescent dyes are widely accepted in various branches of industry including multilayer photoluminescent information recording $[1,2]$, where the quantum yield and relaxation time are most significant values. In this area, the neglect of recording material properties blocked up the development of the most perspective projects, e.g., like a famous $100 \mathrm{~Gb}$ FMD-ROM prototype disc of the "Constellation 3D" [1], where the signal-to-noise ratio and reading rate were found unacceptable.

Luminescent readout signal is spatially isotropic and optical head get just a part of the probing beam energy ( $\alpha=d \Omega / 4 \pi$, where $d \Omega$ is reflected ray recept angle). Luminescence quantum yield $\eta$, absorption factor $k_{A}$, detector system and land exposure loss coefficients ( $k_{R S}$ and $k_{L E}$, respectively) are other reasons of the luminescent signal low power:

$P=P_{0} \eta(d \Omega / 4 \pi) k_{A} k_{R S} k_{L E}$.

It was calculated that the signal power for different registration luminescent materials could be 0.2 to $2.4 \%$ from the power of the reflective disc readout signal. Thus, in this case the problem of low signal-to-noise ratio is a major one, while the main way of its solving is in synthesis of the dye with a high luminescence quantum yield. The luminescence relaxation time, on the other hand, is a main feature of the data reading rate and, therefore, selection of the appropriable registration material will allow getting this parameter up to that of the modern optical discs.

Toward this end, the following organic pyrazoline dyes [3,4] were investigated: 53SM (base pyrazoline UV dye with $5 \%$ addition of polymethylmethacrylat), 59HM (pyrazoline "orange-red" dye with $5 \%$ addition of polymethylmethacrylat), 53SC (pyrazoline UV dye with $5 \%$ addition of polystyrene), 59HC (pyrazoline "orange-red" dye with $5 \%$ addition of polystyrene). Most of them were accepted as a very effective registration media with a quantum yield of luminescence up to $60-70 \%$, the relaxation time less than $100 \mathrm{~ns}$, wide spectrum of the photoluminescence (Fig. 1) and moreover possibility of two-photon absorption. Nevertheless, further research demonstrated a potential for the additional improvement of these characteristics. Our new method is based on the performance of organic dye molecules in the white zeolite matrix. The series of experiments confirmed theoretical consideration that the zeolite submicron pores divide a bulk of the dye into the nanoparticles with a set of improved optical charac- 


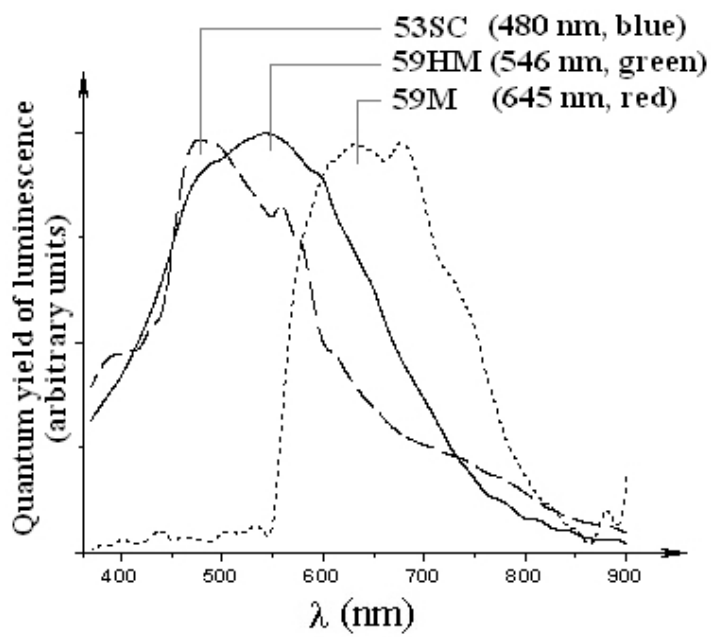

Fig. 1. Pyrazoline dyes luminescent spectra.

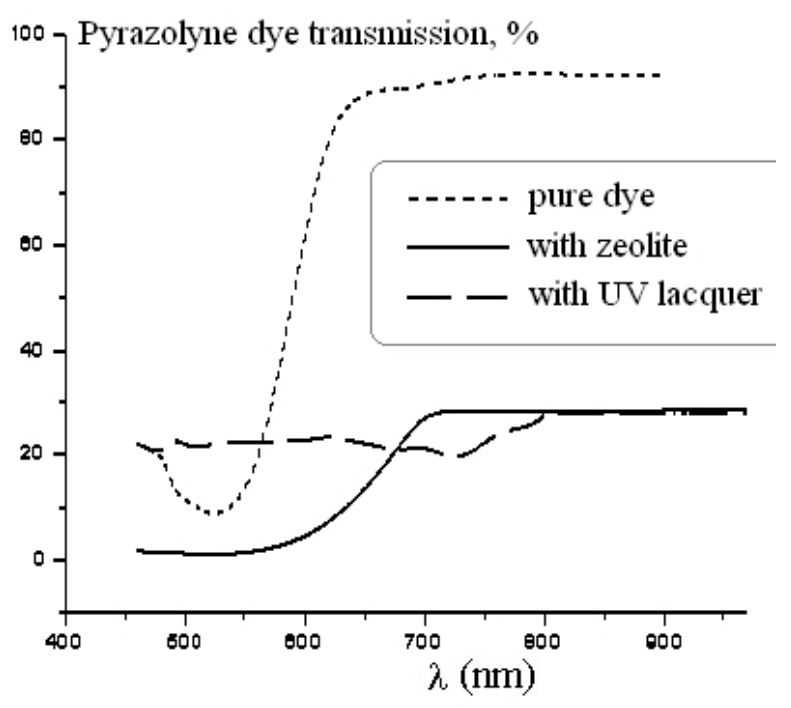

Fig. 2. Transmission ratio of the base pyrazoline "redorange" dye blends with zeolite and UV lacquer.

teristics. And the quantum yield gain is the main of the predicted ones, because nanoparticles of any luminescent material are characterized by the ratio:

$\eta=1 /\left(1+\beta D^{2}\right)$

where $\beta$ is a constant and $D$ is the diameter of nanoparticle.

The main luminescent intensity peak for pyrazoline dyes grows up to $20-30 \%$ (Figs 3,4), but complementary laser annealing increases this parameter at least twice causing complete pores fill with the dye. The growth of the luminescence quantum yield is caused by the quantum size effects that change molecular energy structure of the dye, and thus some forbidden transitions are turning to partially allowed ones. Organic dye absorption growth in this case is also concerned with an

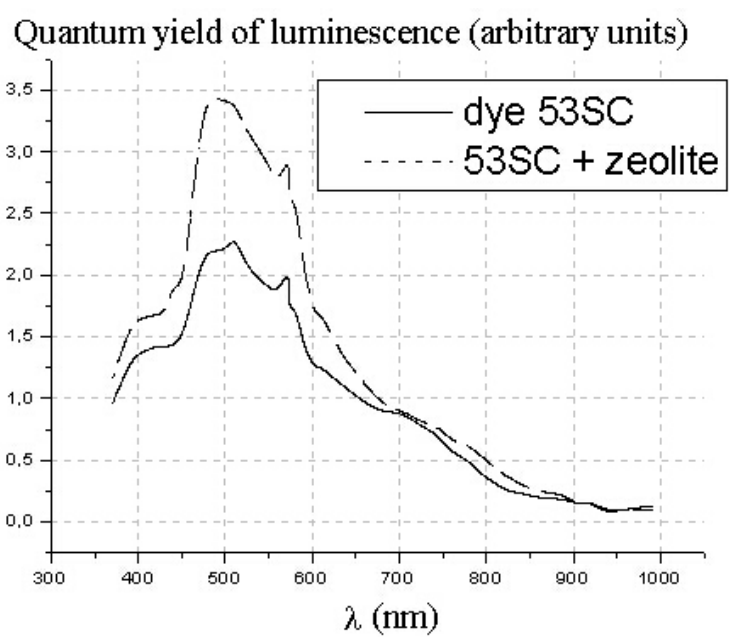

Fig. 3. Pyrazoline dye 53SC (pure and with zeolite) luminescent spectra.

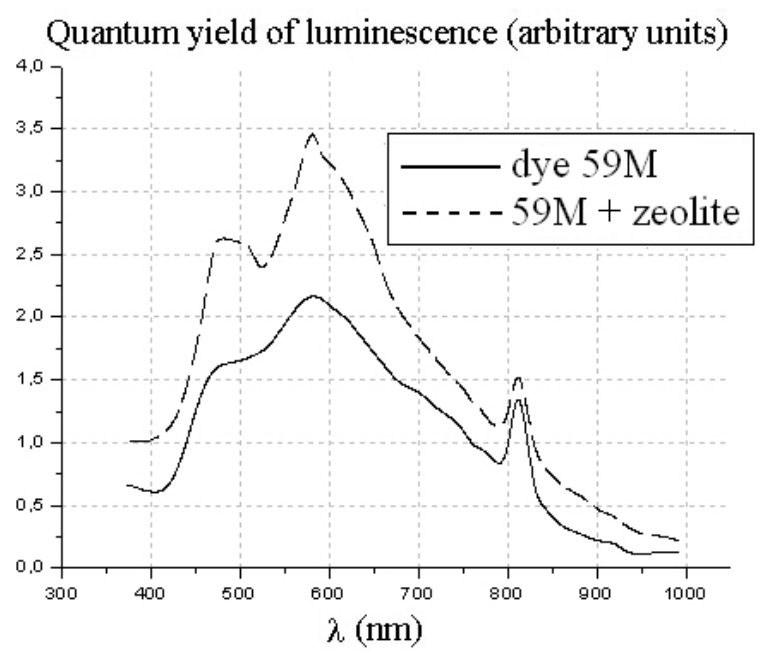

Fig. 4. Pyrazoline dye 59M (pure and with zeolite) luminescent spectra.

appearance of the new allowed transitions. In the zeolite submicron porous structure excited molecules relax to the lower levels and thus absorb larger quantities of the light. Theoretically, these effects can also cause the rise of additional luminescent peaks, which was demonstrated for some types of the pyrazoline dyes. It was investigated that for complex synthesized pyrazoline dyes are crucial $\pi \pi^{*}$ cross-linking with an active energy hydrogen bond with a typical effect of transmission spectrum infrared shift (Fig. 2).

The calculated maximal value of preferred luminescence relaxation time is $\tau_{l}=100 \mathrm{~ns}$. Such a relaxation time value would allow reading data from the luminescent media with a rate equal to multispeed compact disc drive, but for approaching of the rate of DVD or even Blue-Ray Disc drives one must further 
decrease this value. Our experiments showed that the relaxation time value for pyrazoline dyes at the exponential level is $\tau_{l}^{e}=60 \ldots 100 \mathrm{~ns}$ (Fig. 5). Addition of the zeolite decreased the luminescence relaxation time twice $\left(\tau_{l}^{e}=30 \ldots 50 \mathrm{~ns}\right)$, and using the complementary laser annealing decreased this value twice again $\left(\tau_{l}^{e}=15 \ldots 30 \mathrm{~ns}\right)$. Moreover, the complex structure of the luminescence kinetics graph (Fig. 6) shows that pyrazoline has not yet completely filled zeolite pores; it's obvious that $\tau_{l}^{(1)}$ corresponds to the nanoparticle dye structure, while $\tau_{l}^{(2)}$ is the luminescence relaxation time of the bulk registration material part.

Quantum yield of luminescence (arbitrary units)

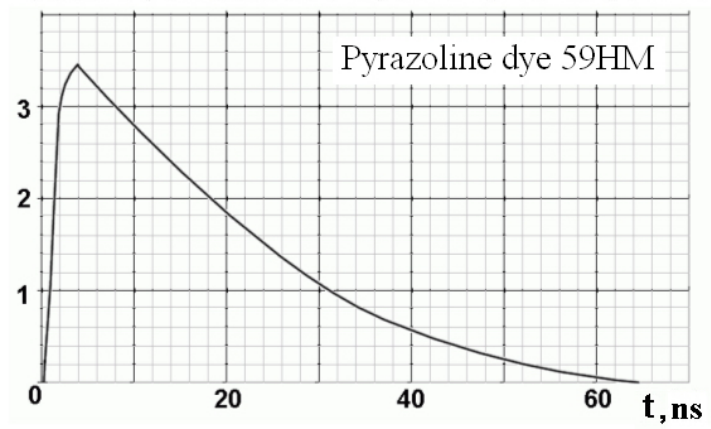

Fig. 5. Pure pyrazoline dye 59HM kinetics of luminescence.

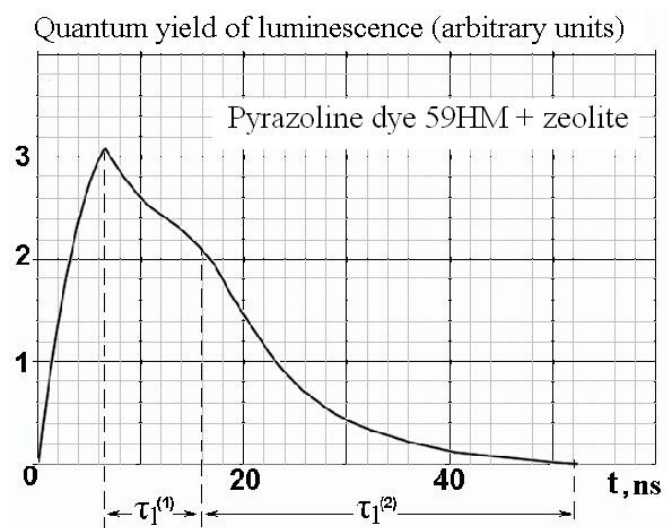

Fig. 6. Pyrazoline dye 59HM with zeolite kinetics of luminescence.
Synthesized pyrazoline dyes also get there application at such a specific area of the information recording as an optical disc counterfeit protection [3-5]. A complex protection procedure includes $1 \mathrm{D}$ and $2 \mathrm{D}$ luminescent barcodes, unreadable sectors zone and luminescent cover with microtext. For plotting all the identification elements, proposed were pyrazoline dyes with additional IR laser light local bleaching. The dyes with zeolite were used in formation of the cover, because this element was developed for visual identification under UV light and material with extremely high quantum yield level of the luminescence could provide the best ergonomics. On the other hand, on the working surface one should use pyrazoline dye with the highest level of transparency for reliability of the readout process.

Thus, synthesis of the luminescent material based on the performance of organic pyrazoline dye molecules in the white zeolite matrix enabled to obtain the favorable abilities in the area of the information recording and security. Theoretical considerations of the high quantum yield and low relaxation time of resultant material got entire experimental confirmation.

\section{References}

1. B.A. Glushko, E.B. Levich, Fluorescent optical memory // United States Patent 6,071,671 G11B 007/24; G11B 007/22, G11B 007/00, p.1-27 (2002).

2. E.V. Beliak, A.A. Kryuchin, A.I. Stetsun, Luminescent readout: the way to increase the capacity of information carriers // Reyestratsiya, zberigannya i obrobka danykh 5(1), p. 3-11 (2003) (in Russian).

3. E.V. Beliak, V.G. Kravets, A.A. Kryuchin, I.A. Kossko, A.A. Pogodina, Application of dyes with luminescent response to creation of registering media and protection of CD against counterfeiting // Ibid. 4(3), p. 15-22 (2003) (in Russian).

4. V.G. Kravets, O.V. Prygun, E.V. Beliak, A.A. Kryuchin, The way to protect compact discs with using a luminescent protective layer // Ukraine Patent 66954, Bulletin No.6, 2004 (in Ukrainian).

5. E.V. Beliak, Technology of deposition of luminescent identifying elements onto the surface of optical disks // Reyestratsiya, zberigannya $i$ obrobka danyh 8(3), p. 3-10 (2006) (in Ukrainian). 